\title{
Toward a global filamentous fungus genome sequencing effort
}

\author{
Nigel Dunn-Coleman and Rolf Prade
}

Last year, "The First Fungal Genome Workshop" was held at the Center for International Trade and Development of Oklahoma State University (OSU; Stillwater, OK). Supported by OSU and several companies representing both industrial biotechnology and the traditional pharmaceutical industries (including Genencor International (Palo Alto, CA), Gist-Brocades (Delft, the Netherlands), Novo Nordisk Biotech (Davis, CA), Schering-Plough (Union, NJ) and Unilever (Vlaardingen, the Netherlands), the workshop was attended by scientists from around the world.

Perhaps the major problem confronting the attendees was which fungus to sequence first. From the industrial biotechnologist's viewpoint, Aspergillus oryzae, Aspergillus niger, Penicillium chysogenum, and Trichoderma reesei are clearly the preferred choices. In terms of medical importance, Aspergillus fumigatus, the causative agent of aspergillosis disease, would seem the logical option. Indeed, it is rumored that the sequencing of this fungus is now underway in at least one genomics company. And the agricultural impact of Aspergillus flavus, which produces an aflatoxin that costs the United States $\$ 100$ million in corn crop loses each year ${ }^{2,3}$, argues strongly that sequencing this genome would pay rich dividends.

Filamentous fungi have also been traditionally economically important as hosts for producing enzymes (e.g., glucoamylase), commodity chemicals (e.g., citrate), and antibiotics (e.g., penicillin). Pharmaceutical manufacturing using these fungi constitutes a $\$ 23$ billion per year industry worldwidie. Fungus-produced antibiotics represent the second largest selling antibiotics, and penicillin production alone accounts for $\$ 4.4$ billion/year worldwide. More recently, several filamentous fungi have been successfully used for heterologous protein production. For example, bovine chymosin expressed in A. niger var. awamori has now received GRAS (generally regarded as safe) status.

Nigel Dunn-Coleman is staff scientist at Genencor International, Palo Alto, CA (NDunn-Coleman@genencor.com) and Rolf Prade is assistant professor at the department of microbiology and molecular genetics, Oklahoma State University, 409 LSE, Stillwater, OK 74078-0289.
On the health front, invasive aspergillosis is an increasingly important opportunistic infection. There has been a 14 -fold increase in its incidence during the past 12 years, as detected by autopsy, and only two drugs are available that are effective in its treatment, neither of which is completely satisfactory. Amphotericin B needs to be given intravenously and has a number of toxic side effects; itraconazole, which can be given orally, is being prescribed imprudently, encouraging the emergence of resistant strains of $A$. fumigatus . $^{4}$

It is not surprising that even the early results of these pilot sequencing efforts are interesting, intriguing, and potentially profitable.

Regrettably, none of these particular fungi has the advantages offered by the two model filamentous fungal systemsAspergillus nidulans (31.0 Mb of DNA) and Neurospora crassa (42.0 Mb) - namely, more than 50 years of classical genetic studies and biochemical analysis. Therefore, the decision to convene a workshop, organized by Joan Bennett at Tulane University (New Orleans, LA) to begin pilot genomic sequencing projects for both $N$. crassa and A. nidulans, makes good scientific sense. It also makes sense from the industrial, medical, and agricultural viewpoints.

Along these lines, a pilot project to sequence the $2.9 \mathrm{Mb}$ chromosome IV of $A$. nidulans is to be supported by a unique industry-academia consortium. Industrial participants are Genencor International, Novo Nordisk Biotech, Gist-Brocades, Glaxo Wellcome (London), Ammano (Tsukuba, Japan), and Kikkoman (Chiba, Japan). Sequencing will take place at Texas A\&M University (College Station, TX) and sequence assembly at OSU, which will also be responsible for maintaining a public domain Web site containing all the sequence information. Very recently, a proposal has also been submitted to the European Union to sequence the $3.5 \mathrm{Mb}$ chromosome III of $A$. nidulans.
It is not surprising that even the early results of these pilot sequencing efforts are interesting, intriguing, and potentially profitable. For example, at last year's meeting for the Society for Industrial Microbiology in Reno, NV, Doris Kupfer, working with Bruce Roe's group at the University of Oklahoma (Tulsa, OK) reported that almost $40 \%$ of the expressed sequence tags (ESTs) they have sequenced from $A$. nidulans have no matches in any of the genome databases. And perhaps more importantly, nearly $7 \%$ of the remaining ESTs have no homology to yeast, but do have homologs in the human genome $e^{5}$.

What is surprising, given the above, is that, to date, no major commitment for fungal genome sequencing has come from any North American, European, or Asian government. But even if one assumes that over the next several years the genomes of $A$. nidulans and $N$. crassa are completely sequenced, and perhaps one or two plant pathogenic fungi as well, are the tools to perform functional genomics with these fungi being developed?

Consider that the efforts of a large Saccharomyces cerevisiae researcher community and many decades of research only identified half the genes of this organism by classical and molecular methods. The response of the yeast community has been to establish a global effort to analyze the function of open reading frames (ORFs) with no discernible function. The technologies to be employed include gene expression analysis via hybridization array technology, deletion analysis, protein localization, and metabolic control analysis ${ }^{6,7}$.

In the cases of $N$. crassa and A. nidulans, between $30 \%$ and $50 \%$ of the ESTs sequenced so far appear to have no homologies in any of the genome databases, and the industrially, medically, and agriculturally important fungi are not as amenable to genetic analysis as $S$. cerevisiae. Thus, developing new tools-as well as adapting molecular tools being used to extend the functional analysis of genes in yeast-must of necessity become a priority if expanded sequencing efforts are to pay their anticipated, significant returns.

\footnotetext{
1. Hammer, L. 1997. Fungal Genet. Biol. 21:8-10.

2. Bennett, J. 1997. Fungal Genet. Biol. 21:3-7.

3. Aramayo, R. and Bennett, J. 1997 ASM News 63:176-177.

4. Denning, D.W. et al. 1997. Antimicrob. Agents Chemother. 41:1364-1368.

5. http://fungus.genetics.uga.edu

6. Winzeler, E. 1997. ASM News 63:312-317.
} 